Review

\title{
Clinical Application of High-intensity Focused Ultrasound in Cancer Therapy
}

\author{
Yi-Hsuan Hsiao ${ }^{1,2}{ }^{\bowtie}$, Shou-Jen Kuo ${ }^{3}$, Horng-Der Tsai ${ }^{2}$, Ming-Chih Chou ${ }^{1}$, Guang-Perng Yeh ${ }^{1,2}{ }^{\bowtie}$ \\ 1. School of Medicine, Chung Shan Medical University, Taichung, Taiwan \\ 2. Department of Obstetrics and Gynecology, Changhua Christian Hospital, Changhua, Taiwan \\ 3. Comprehensive Breast Cancer Center, Changhua Christian Hospital, Changhua, Taiwan \\ $\square$ Corresponding authors: Yi-Hsuan Hsiao, M.D., PhD. School of Medicine, Chung Shan Medical University, Department of Obstetrics and Gynecology, \\ Changhua Christian Hospital, 135 Nanhsiao Street, Changhua, 500 Taiwan. Phone: +886-4-7238595 Fax: +886-4-7228289 E-mail: 54315@cch.org.tw. Or \\ Guang-Perng Yeh, M.D. School of Medicine, Chung Shan Medical University, Department of Obstetrics and Gynecology, Changhua Christian Hospital, 135 \\ Nanhsiao Street, Changhua, 500 Taiwan. Phone: +886-4-7238595 Fax: +886-4-7228289 E-mail: 4011@cch.org.tw
}

() Ivyspring International Publisher. Reproduction is permitted for personal, noncommercial use, provided that the article is in whole, unmodified, and properly cited. See http://ivyspring.com/terms for terms and conditions.

Received: 2015.09.18; Accepted: 2015.12.03; Published: 2016.01.03

\begin{abstract}
The treatment of cancer is an important issue in both developing and developed countries. Clinical use of ultrasound in cancer is not only for the diagnosis but also for the treatment. Focused ultrasound surgery (FUS) is a noninvasive technique. By using the combination of high-intensity focused ultrasound (HIFU) and imaging method, FUS has the potential to ablate tumor lesions precisely. The main mechanisms of HIFU ablation involve mechanical and thermal effects. Recent advances in HIFU have increased its popularity. Some promising results were achieved in managing various malignancies, including pancreas, prostate, liver, kidney, breast and bone. Other applications include brain tumor ablation and disruption of the blood-brain barrier. We aim at briefly outlining the clinical utility of FUS as a noninvasive technique for a variety of types of cancer treatment.
\end{abstract}

Key words: focused ultrasound surgery, cancer therapy, high-intensity focused ultrasound, ablation

\section{Introduction}

The treatment of cancer is an important issue in both developing and developed countries. Clinical use of ultrasound in cancer is not only for the diagnosis but also for the treatment. Use of focused ultrasound in experimental biology and the first therapeutic trial was carried out in 1942 (1). The use of therapeutic ultrasound for cancer treatment was established by the 1970s (2). Focused ultrasound surgery (FUS) is a noninvasive technique. By using the combination of high-intensity focused ultrasound (HIFU) and imaging method, FUS has the potential to ablate tumor lesions precisely. Recent advances in HIFU have increased its popularity. HIFU is used to manage a variety of tumors and palliative care $(3,4)$.

Higher energy and intensity are used in therapeutic purpose than in diagnostic Ultrasound (US) though HIFU principles are the same as conventional US (5). The main mechanisms of HIFU ablation in- volve mechanical and thermal effects (Figure 1). The local tissue temperature could be elevated to higher than $60^{\circ} \mathrm{C}$ by the thermal effect. The thermal effect causes tumor cell destruction via coagulation necrosis in HIFU therapy. The mechanical effects include micro-streaming, radiation force and cavitation.

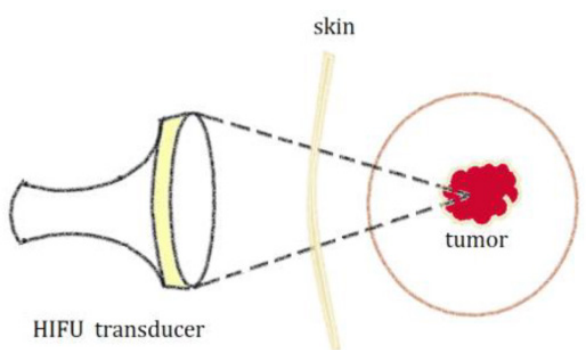

Figure 1. Diagram for the principle of High Intensity Focused Ultrasound (HIFU) for tumor treatment 
There are three main categories of HIFU devices for clinical use, including extracorporeal, interstitial and trans-rectal. Extracorporeal probes are used to target tumor lesions through the skin, trans-rectal instrument are designed to treat prostate tumors and interstitial devices are used to treat esophageal tumor and tumor of biliary duct (4).

Clinical HIFU procedures are generally combined with magnetic resonance imaging (Magnetic Resonance-guided Focused Ultrasound (MRgFUS)) or ultrasound imaging (Ultrasound-guided HighIntensity Focused Ultrasound (USgHIFU or USgFUS)) as image guidance and treatment monitoring. MRgFUS technology combines Magnetic Resonance Imaging system (MRI) which provides three dimension anatomy for precise tumor targeting, continuously monitoring the tissue effect for controlled treatment and post treatment contrast imaging for accurate conduct validation and a HIFU beam which destroys the diseased tissue by a non-invasive procedure. The MRgFUS procedure in clinical manifestation was established for the treatment of symptomatic benign uterine tumor and palliative treatment for patients with painful bone metastases (6). Recent advances in HIFU have increased its popularity. Some promising results were achieved in managing various malignancies, including pancreas, prostate, liver, kidney, breast and bone (7-12). Other applications include brain tumor ablation and disruption of the blood-brain barrier (6).

We aim at briefly outlining the clinical utility of FUS as a noninvasive technique for a variety of types of cancer treatment.

\section{Clinical Application of HIFU in Cancer Therapy}

\section{Prostate cancer}

The treatment of prostate cancer is dependent on stage, Gleason score, PSA level and patient age (13). Besides radical prostatectomy (RP), there are many alternative local treatment options as follows: external-beam radiation therapy (EBRT), and/ or brachytherapy, cryosurgical ablation and HIFU technology (13).

The HIFU technique could be an alternative to surgery especially for patients with comorbidity (4). Careful patient selection is important for prognosis. The patients with favorable pathologic Gleason score and lower pre-HIFU PSA seem to present better outcomes (14). Warmuth et al. conducted a review of prostate cancer treatment with HIFU systems. Twenty uncontrolled prospective case series were identified. The authors showed overall survival rate was $83 \%$ and cancer-specific survival rate was $98 \%$ at 8 years
(15).

A recent clinical study included 918 patients treated with Sonablate ${ }^{\circledR}$ (SB) devices for localized prostate cancer. The results showed the cancer specific survival rate was $97.4 \%$ and ten year overall survival rate was $89.6 \%$. The authors concluded that technical, imaging and technological advancements improved clinical outcomes of patients with HIFU therapy (7). Adverse effects, including urethral stricture (19.7\%), epididymitis $(6.2 \%)$, urinary incontinence $(2.3 \%)$ and rectourethral fistula $(0.1 \%)$, were concerned in their series (7). However, current data is of low quality for proving the safety and efficacy of prostate cancer treatment using HIFU systems $(15,16)$. Current data from HIFU are not extensive enough for prostate cancer treatment recommendations (13). More research on clinical utility of HIFU in prostate cancer is needed for the widespread use of HIFU $(15,16)$.

\section{Breast cancer}

The current clinical management of localized breast cancer is breast-conserving therapy (17). For advancement of technology, imaging and improvement of adjuvant therapy, the surgical management of breast cancer has progressed. Noninvasive ablation of breast cancers could be an alternative option for small breast tumors (8). However, many questions arise in clinical implementation of imaging-guided percutaneous ablation of breast cancer. These questions and issues should be addressed before noninvasive percutaneous ablation can be adopted as an alternative treatment of early breast cancer (18).

MR-guided high-intensity focused ultrasound, which offers precision of anatomy and temperature mapping for ablation of a target tissue without causing skin or subcutaneous tissue disruption in the path of focused ultrasound beam, is completely non-invasive for breast cancer therapy. However, both clinical and technical challenges have to be solved before MRgFUS can be adopted as a routine clinical procedure (17).

Many clinical studies on HIFU treatment of breast cancer were reported (19-24). Wu et al. showed complete necrosis of tumors cells by HIFU technique and concluded that HIFU system could be effective in localized breast cancer treatment (22-24). The authors proposed that more clinical trials should be conducted for determining the future role of HIFU system although it seems safe and effective in their clinical study (23). Li et al. reviewed 11 arms of breast cancer treatment using HIFU guided by US or MRI during the period 2002-2010. There were a total of 173 patients treated with HIFU therapy and the tumor extent varied from $0.5 \mathrm{~cm}$ to $6.0 \mathrm{~cm}$ diameter. Complete ablation rate was $71 \%(123 / 173)$ by MRI-guided HIFU or 
US-guided HIFU therapy. The complete necrosis rates were $59 \%(71 / 121)$ by MRI-guided HIFU and $96 \%$ (50/52) by US-guided HIFU therapy (25).

There are several advantages of HIFU therapy for breast cancer treatment, including preserving the structure and function of breast, no bleeding, no scaring and no radiation. However, there are disadvantages (limitations) of HIFU therapy for breast cancer treatment. $\mathrm{Li} \mathrm{A}$ and $\mathrm{Wu} \mathrm{PH}$ proposed three major problems with the HIFU therapy: (1) It is difficult to confirm whether ablation margin is free; (2) Recurrence concern exists in 5 multifocal or multi-centric breast cancer after HIFU treatment; (3) necrotic masses remaining in the breast after HIFU therapy could cause additional psychological burden to the patient.

A recent study of systematic review of HIFU ablation for breast cancer treatment showed a lack of report for consistent histopathology (26). Using HIFU therapy for breast cancer treatment is not common at the present time, and efforts to promote wider application of HIFU may be expected in the future (25). For large, prospective clinical trials to evaluate HIFU ablation, it is necessary to have consistent lesion histopathology and imaging follow-up for margin necrosis (26).

\section{Liver cancer}

Liver transplantation or surgical resection has been the gold standard management for liver cancer. The successful focused extracorporeal pyrotherapy was reported in 1993 (27). However, there are many challenges for extracorporeal ablation of liver tissue by HIFU, including respiratory movement of the liver, beam propagation through the ribs, and long ablation times due to huge tumors $(28,29)$. HIFU technology has been driven to overcome the challenges by several studies during the following decade.

$\mathrm{Wu}$ et al. evaluated the efficiency using the combination of USgHIFU modality and trans-catheter arterial chemoembolization (TACE) for stage IVA liver cancer treatment by a randomized study including 50 patients with stage IVA liver cancer.

Median survival time was significantly longer in those treated with combined HIFU and TACE procedures compared to those with TACE performed alone (11.3 months vs. 4 months; $\mathrm{p}=0$. 004). The six-month survival rate was $80.4 \%-85.4 \%$ for patients treated combined HIFU and TACE therapy and $13.2 \%$ in patients with TACE performed alone $(\mathrm{P}=.002)(30)$. A study determined long-term effects and major prognostic factors for 73 unresectable hepatocellular carcinoma patients treated with combined HIFU and TACE. The results showed $45 \%$ of patients achieved complete ablation. The authors concluded that major prognostic factors included tumor size and ablation response (31).

For HIFU treatment alone, $\mathrm{Ng} \mathrm{KK}$ et al conducted a study which included 49 patients receiving a session of HIFU modality with a curative intent for unresectable liver cancer. The results showed the primary effectiveness rate $79.5 \%$ (39 of 49 patients), hospital mortality rate $2 \%(\mathrm{n}=1)$ and the complication rate $8.1 \%(\mathrm{n}=4)$. The authors concluded that HIFU treatment was effective for unresectable hepatocellular carcinoma and patients had favorable survival outcome (32).

Safety of HIFU procedures is a concern. The complications of HIFU ablation for hepatic cancer patients have been reported, including biliary obstruction, pleural effusion, pneumothorax, fistula formation, diaphragmatic rupture and rib fracture (33). Furthermore, the safety of HIFU ablation for liver cancer adjacent to major blood vessels was assessed by evaluating hepatic vein branches, inferior vena cava, and the portal vein and its branches in 39 patients with 42 tumors which are adjacent to $1 \sim 4$ main vessels. No large blood vessel injury was detected among the 42 tumors. HIFU therapy accomplishing complete tumor necrosis even for lesions adjacent to major vessels was demonstrated (34).

Complications of HIFU therapy for patients with liver cancer include skin burns, pain, induced fever, mild impairment of liver function or renal function and local damage (35). The local and systemic complication should be a concern even though

HIFU is a minimally invasive technique. HIFU ablation of liver tumor could be accurate by intra-operative assessment of treatment providing precise measurement of ablation zone. The effect was demonstrated by MRI follow-up, which correlated with intra-operative estimates of treatment volume (36).

The feasibility and efficacy of HIFU therapy for inoperable liver cancer was demonstrated by a recent clinical study. 116 primary liver cancer and 71 metastatic liver cancer cases were treated with HIFU; complete response and partial response were 55 patients $(29.4 \%)$ and 73 patients $(39.0 \%)$, respectively. The authors showed that HIFU is feasible in liver cancer treatment and the efficiency of HIFU ablation may be improved by repeated treatment or increasing treatment times (9).

The long term and short term efficacy of HIFU therapy in advanced liver cancer was shown by a clinical study in patients with surgically unrespectable hepatocellular carcinoma. The authors demonstrated that no severe complications occurred during and after HIFU and concluded that HIFU is a safe ablation therapy for patients with advanced hepato- 
cellular carcinoma (37). A recent study analyzed HIFU ablation for 187 patients with inoperable liver cancer and showed response rates $90.5 \%$ in left lobe cancer and $64.1 \%$ in right lobe cancer (9).

\section{Kidney cancer}

Renal cancers are currently diagnosed at earlier stage and smaller overall size than previously $(38,39)$. The great majority of renal cell tumors were found incidentally; $61 \%$ of patients diagnosed with kidney cancer were asymptomatic (40). Among nephron-sparing surgery options, partial nephrectomy $(\mathrm{PN})$ is the treatment of choice for peripheral lesions (41). Nevertheless, surgical complication rates ranging from $9 \%$ to $33 \%$ was reported, especially for those patients with medical co-morbidities (42). New alternative minimally invasive nephron-sparing treatments of kidney cancer are desirable.

There are several ablation treatment modalities in the clinical management of renal cell carcinoma, including radiofrequency ablation, HIFU ablation, and cryoablation (10). Ablative therapies were recommended in patients unfit for surgery, or in patients not considered candidates for elective surveillance, or patients with small renal masses (10).

Surgery remains the standard treatment for localized kidney cancer at the present time. However, the incidence of small kidney cancer increases and the surgery has shifted toward nephron sparing treatment. Minimally invasive ablation therapy presents an alternative clinical management of kidney cancer (43). Extracorporeal HIFU therapy results in 'trackless' homogenous tissue ablation is entirely noninvasive (43). The outcomes associated with ablative modalities could be improved with advanced technology and the applications could be expanded with more evidence of long-term efficacy (10).

Extracorporeal HIFU treatment for renal tumors is well tolerated but has to be considered an experimental approach (44). A recent clinical study comprising 17 patients underwent extracorporeal HIFU ablation and monitored by Real-time diagnostic ultrasonography. The results showed that stable lesions in two-thirds of patients. HIFU ablation could be a treatment option for selected cases (43).

A clinical study demonstrated that laparoscopic HIFU for renal tumors could be feasible, and achieved homogenous ablation with low associated morbidity (45).

HIFU therapy for kidney cancer patients was presented by $\mathrm{Wu}$ et al., where 12 cases of advanced stage kidney cancer and 1 case of colon cancer with kidney metastasis were included. Among the 13 patients, 7 died (median survival 14.1 months) and 6 were still alive (median follow-up of 18.5 months).
HIFU could be feasible and safe for select patients with advanced stage renal malignancy (46).

\section{Pancreatic cancer}

More than $80 \%$ of pancreatic cancer patients present advanced disease, and are inappropriate for surgical treatment. Standard treatment options available for unresectable pancreatic cancer include chemotherapy, radiotherapy or a combination of the two (11). Prognosis in pancreatic cancer is extremely poor. Pain relief and palliative treatment for local tumor control are the aims of treatment for those patients with inoperable pancreatic cancer (29). HIFU ablation has been used as primary and palliative treatment for pancreatic cancer (39). Furthermore, HIFU ablation has been designed as an additional treatment with tumor debunking (11). Initial studies implied that ablative therapies may confer a survival benefit over supportive care (47). The advantages of HIFU ablation include less hurt and short recovery time. Ultrasound-guided HIFU ablation as a feasible procedure to manage solid tumors was demonstrated by a clinical study which included 38 lesions in difficult locations of the liver and pancreas (48).

A clinical study of HIFU comprised 89 advanced pancreatic cancer patients who underwent HIFU therapy. The results showed the median survival of 26.0 months for stage II, 11.2 months for stage III and 5.4 months for stage IV cancer patients. Pain relief achieved $80.6 \%$ of patients (49). Clinical application of HIFU modality is promising for palliation of pain related to pancreatic cancer (49). Wu et al. reported an initial experience of US-guided HIFU treatment in eight patients with advanced- stage pancreatic cancer. The authors demonstrated that preexisting severe back pain disappeared after HIFU therapy and no complications were observed in the patients. HIFU ablation could be a safe procedure for patients with advanced pancreatic cancer (50).

HIFU approach has been shown safe and feasible, effectively remitting cancer-related abdominal pain, when used alone or combined with chemotherapy for locally advanced pancreatic cancer patients (11). If the long-term efficacy data is favorable in large randomized clinical trials, HIFU therapy could be a treatment option for pancreatic cancer patients (11). Furthermore, new applications of HIFU therapy may improve outcomes of pancreatic cancer patients, through enhancing drug delivery to the tumor or inducing host immunity to both local and metastatic tumors (51).

\section{Primary and secondary bone malignancy}

Primary bone malignancies are rare and occur most frequently in young adults and children. Sur- 
gery and radiation are common options for management of bone tumors (4). Local unresectable recurrence of osteosarcoma is challenging to treat. HIFU technology has the potential to ablate and inactive tumors. Limb sparing is a potential advantage of HIHU therapy for bone malignancies. Yu et al. conducted a clinical study of HIFU therapy for 27 patients with local unresectable bone malignancies. The results showed 4 progression, 9 stable, 2 complete response and 12 partial response after HIFU therapy. HIFU modality is safe for managing local unresectable recurrence of bone tumor treatment (12).

A study showed the efficiency of HIFU in clinical application of 25 malignant bone tumor patients, which were evaluated by biochemical markers and imaging systems (magnetic resonance imaging or positron emission tomography-computed tomography) before and after HIFU therapy. The results showed that biochemical markers were improved and no severe complications were noted. For primary bone tumor patients, the response rate achieved $84.6 \%$; for patients with metastatic bone tumors, the response rate was $75.0 \%$. The authors concluded that HIFU ablation seems to be successful in managing primary bone malignancies (52). However, a different opinion was expressed for concern of survival, which insists HIFU is not indicated for treatment of primary bone sarcomas (53).

A multicenter study investigated MRgFUS surgery for palliative care in patient with bone metastases. The results showed $72 \%(18 / 25)$ of the patients with significant pain improvement and no occurrence of device-related adverse effect. MRgFUS could be an option for palliative treatment in patients suffering from painful bone metastases (54).

Gianfelice et al. showed that MRgFUS technique provided palliative treatment of bone metastases with little or no morbidity (55). Preliminary clinical experience of MRgFUS for palliative treatment of pain in thirteen patients with bone metastases was demonstrated. Twelve patients received adequate treatment: two patients died from disease progression and ten patients had prolonged improvement in pain score. MRgFUS may be an alternative for bone metastasis pain relief (56).

\section{Trans-cranial HIFU therapy for brain tumor}

The major obstacle for HIFU technology in brain tumor therapy is skull bone as the focused US field is disturbed and most of the US energy is absorbed by the skull bone. These difficulties have been overcome for the development of adaptive focusing techniques and large-sized phased array US transducers. Trans-cranial MRgFUS has been established and the clinical potential applications are researched in a fast-growing field of neuropathic pain and brain tumor therapy (57). MRgFUS system may be used to temporarily disrupt the blood-brain barrier for drug delivery to brain tumors (58).

A pilot study included 15 patients who underwent MRgFUS for the treatment of medication-refractory essential tremor. The results showed total tremor scores improved from 54.9 to 24.3 $(\mathrm{P}=0.001)$ (59). The application of MRgFUS in 13 patients with chronic Parkinson's disease was reported. The authors showed the accuracy, safety and feasibility of the MRgFUS pallidothalamic tractotomy (60).

The clinical feasibility of trans-cranial MRgFUS was evaluated by McDannold et al. (61) Three glioblastoma patients underwent this procedure of multiple focused ultrasound exposures. The authors demonstrated the beam from HIFU at the brain with the magnetic resonance temperature imaging. However, MRgFUS seemed not to achieve thermal coagulation $(61,62)$.

Trans-cranial MRgFUS could be a treatment option for brain diseases (62). HIFU modality for functional neurosurgery was shown by a clinical study where 9 patients suffering from chronic neuropathic pain were included and all treatments were all well tolerated. The results show the helpful clinical utility of trans-cranial HIFU modality for brain functional diseases (62). Large randomized clinical trials are needed to assess the safety and efficacy of trans-cranial HIFU therapy for brain tumor and neuropathic pain.

\section{Localized drug and gene delivery mediated by ultrasound}

Localized drug and gene delivery mediated by ultrasound is a potential beneficial cancer treatment $(63,64)$. Low frequency ultrasound (LFUS) was the leading method of downsizing multilamellar vesicles into small unilamellar vesicles in the past. Localized and controlled drug released from liposomes by using ultrasound has been shown recently (64). Heat-sensitive microbubble agent (HSM) as a contrast agent for intra-operative assessment of cancer ablation margins was proposed (65).

Acoustic cavitation plays a key role in a wide array of therapeutic ultrasound.

There are several types of cavitation activity which serves to accelerate heating

(66). The activation of heat-sensitive trans-gene by HIFU using a tumor model was proposed and the authors suggest the possibility of applying HIFU ablation with heat- regulated gene therapy for cancer (67). Hancock et al. investigated pulsed HIFU-enhanced delivery by pulsed-HIFU exposures to the muscle and administrating a variety of fluoro- 
phores. The exposure resulted in some structural alterations between the muscle fiber bundles and the influence consisted with increase of tissue permeability $(68)$.

Huber et al. demonstrated that focused ultrasound modality provided an improvement of DNA plasmid transferred in vivo and in vitro. The study showed a favorable system for gene-based medical treatments with spatial regulation by focused ultrasound (69). Plathow et al. demonstrated focal gene induction into a parenchymal organ in rats by HIFU technique under the control of MRI (70).

The roles of ultrasound and magnetic resonance have been demonstrated in delivering local drugs. MRI presents entry of imaging biomarkers and provides real-time temperature mapping. Ultrasound can be used for the local drug delivery by carriages. Ultrasound contrast agents could enhance effects by cavitations (71).

\section{Conclusion}

Whether HIFU should be used as a clinical routine treatment modality is dependent on the following factors: clinical outcome, feasibility, therapy device, efforts and prices. HIFU technology can be improved by using micro-bubble contrast agents or can be used to transfer genes or drugs for future oncological HIFU applications. HIFU technique should be monitored and tested for safe operation and quality assurance. The clinical applications of HIFU are continuing to improve and expand but HIFU has to be improved to make it more widely adopted. Present clinical results are encouraging and several ongoing studies will provide more clinical data that will be important for the safe and effective use of HIFU for cancer treatment and also as a gene or drug delivery method.

\section{Competing Interests}

The authors have declared that no competing interest exists.

\section{References}

1. Lynn JG, Zwemer RL, Chick AJ, Miller AE. A new method for the generation and use of focused ultrasound in experimental biology. J Gen Physiol. 1942; 26: $179-93$.

2. Kremkau FW. Cancer therapy with ultrasound: a historical review. J Clin Ultrasound. 1979; 7: 287-300.

3. Miller DL, Smith NB, Bailey MR, et al. Overview of therapeutic ultrasound applications and safety considerations. J Ultrasound Med. 2012: 31: 623-34.

4. Di Mari A, Rametta Giuliano S, Lanteri E, et al. Clinical use of high-intensity focused ultrasound in the management of different solid tumors. WCRJ 2014; 1: 295

5. Zhou YF. High intensity focused ultrasound in clinical tumor ablation. World J Clin Oncol. 2011; 2: 8-27.

6. Trumm CG, Napoli A, Peller M, et al. MR-guided focused ultrasound. Current and future applications. Radiologe. 2013. 53: 200-8.

7. Uchida T, Tomonaga T, Kim H, et al. Improved outcomes with advancements in high intensity focused ultrasound devices for the treatment of localized prostate cancer. J Urol. 2015; 193: 103-10.

8. Sabel MS. Nonsurgical ablation of breast cancer: future options for small breast tumors. Surg Oncol Clin N Am. 2014; 23: 593-608.
9. Chen L, Wang $\mathrm{K}$, Chen $\mathrm{Z}$, et al. High intensity focused ultrasound ablation for patients with inoperable liver cancer. Hepatogastroenterology. 2015; 62: 140-3.

10. Klatte T, Kroeger N, Zimmermann U, Burchardt M, Belldegrun AS, Pantuck AJ. The contemporary role of ablative treatment approaches in the management of renal cell carcinoma (RCC): focus on radiofrequency ablation (RFA), high-intensity focused ultrasound (HIFU), and cryoablation. World J Urol. 2014; 32: 597-605.

11. Wu F. High intensity focused ultrasound: a noninvasive therapy for locally advanced pancreatic cancer. World J Gastroenterol. 2014; 20: 16480-8.

12. Yu W, Tang L, Lin F, Yao Y, Shen Z, Zhou X. High-intensity focused ultrasound: Noninvasive treatment for local unresectable recurrence of osteosarcoma. Surg Oncol. 2015; 24: 9-15.

13. Heidenreich A, Bastian PJ, Bellmunt J, et al. EAU guidelines on prostate cancer. part 1: screening, diagnosis, and local treatment with curative intent-update 2013. Eur Urol. 2014; 65: 124-37.

14. Alkhorayef M, Mahmoud MZ, Alzimami KS, Sulieman A, Fagiri MA. High-Intensity Focused Ultrasound (HIFU) in Localized Prostate Cancer Treatment. Pol J Radiol. 2015; 80: 131-41.

15. Warmuth M, Johansson T, Mad P. Systematic review of the efficacy and safety of high-intensity focused ultrasound for the primary and salvage treatment of prostate cancer. Eur Urol. 2010; 58: 803-15.

16. Lukka H, Waldron T, Chin J, et al. Genitourinary Cancer Disease Site Group of Cancer Care Ontario's Program in Evidence-Based Care. High-intensity focused ultrasound for prostate cancer: a systematic review. Clin Oncol (R Coll Radiol). 2011; 23: 117-27.

17. Merckel LG, Bartels LW, Köhler MO, et al. MR-guided high-intensity focused ultrasound ablation of breast cancer with a dedicated breast platform. Cardiovasc Intervent Radiol. 2013; 36: 292-301.

18. Fornage BD, Hwang RF. Current status of imaging-guided percutaneous ablation of breast cancer. AJR Am J Roentgenol. 2014; 203: 442-8.

19. Huber PE, Jenne JW, Rastert R, et al. A new noninvasive approach in breast cancer therapy using magnetic resonance imaging-guided focused ultrasound surgery. Cancer Res. 2001; 61: 8441-7.

20. Hynynen K, Pomeroy O, Smith DN, et al. MR imaging-guided focused ultrasound surgery of fibroadenomas in the breast: a feasibility study. Radiology. 2001; 219: 176-85.

21. Furusawa $\mathrm{H}, \mathrm{Namba} \mathrm{K}, \mathrm{Nakahara} \mathrm{H}$, et al. The evolving non-surgical ablation of breast cancer: MR guided focused ultrasound (MRgFUS). Breast Cancer. 2007; $14: 55-8$

22. Wu F, Wang ZB, Cao YD, et al. "Wide local ablation" of localized breast cancer using high intensity focused ultrasound. J Surg Oncol. 2007; 96: 130-6.

23. Wu F, Wang $\mathrm{ZB}$, Zhu $\mathrm{H}$, et al. Extracorporeal high intensity focused ultrasound treatment for patients with breast cancer. Breast Cancer Res Treat. 2005; 92: 51-60.

24. Wu F, Wang ZB, Cao YD, et al. A randomized clinical trial of high-intensity focused ultrasound ablation for the treatment of patients with localized breast cancer. Br J Cancer. 2003; 89: 2227-33.

25. $\mathrm{Li} \mathrm{S}, \mathrm{Wu} \mathrm{PH}$. Magnetic resonance image-guided versus ultrasound-guided high-intensity focused ultrasound in the treatment of breast cancer. Chin J Cancer. 2013; 32: 441-52.

26. Peek MC, Ahmed M, Napoli A, et al. Systematic review of high-intensity focused ultrasound ablation in the treatment of breast cancer. Br J Surg. 2015; 102: $873-82$

27. Vallancien G, Chartier-Kastler E, Harouni M, Chopin D, Bougaran J. Focused extracorporeal pyrotherapy: experimental study and feasibility in man. Semin Urol. 1993; 11: 7-9.

28. Sibille A, Prat F, Chapelon JY, et al. Extracorporeal ablation of liver tissue by high-intensity focused ultrasound. Oncology. 1993; 50: 375-9.

29. Malietzis G, Monzon L, Hand J, et al. High-intensity focused ultrasound: advances in technology and experimental trials support enhanced utility of focused ultrasound surgery in oncology. Br J Radiol. 2013; 86: 20130044.

30. Wu F, Wang ZB, Chen WZ, et al. Advanced hepatocellular carcinoma: treatment with high-intensity focused ultrasound ablation combined with transcatheter arterial embolization. Radiology. 2005; 235: 659-67.

31. Jin C, Zhu H, Wang Z, et al. High-intensity focused ultrasound combined with transarterial chemoembolization for unresectable hepatocellular carcinoma: long-term follow-up and clinical analysis. Eur J Radiol. 2011; 80: 662-9.

32. $\mathrm{Ng} \mathrm{KK}$, Poon RT, Chan SC, et al. High-intensity focused ultrasound for hepatocellular carcinoma: a single-center experience. Ann Surg. 2011; 253: 981-7.

33. Jung SE, Cho SH, Jang JH, Han JY. High-intensity focused ultrasound ablation in hepatic and pancreatic cancer: complications. Abdom Imaging. 2011; 36: 185-95.

34. Zhang L, Zhu H, Jin C, et al. High-intensity focused ultrasound (HIFU): effective and safe therapy for hepatocellular carcinoma adjacent to major hepatic veins. Eur Radiol. 2009; 19: 437-45.

35. Li JJ, Gu MF, Luo GY, Liu LZ, Zhang R, Xu GL. Complications of high intensity focused ultrasound for patients with hepatocellular carcinoma. Technol Cancer Res Treat. 2009; 8: 217-24.

36. Leslie $\mathrm{T}$, Ritchie R, Illing $\mathrm{R}$, et al. High-intensity focused ultrasound treatment of liver tumours: post-treatment MRI correlates well with intra-operative estimates of treatment volume. Br J Radiol. 2012; 85: 1363-70.

37. Li YY, Sha WH, Zhou YJ, Nie YQ. Short and long term efficacy of high intensity focused ultrasound therapy for advanced hepatocellular carcinoma. J Gastroenterol Hepatol. 2007; 22: 2148-54. 
38. Kane CJ, Mallin K, Ritchey J, Cooperberg MR, Carroll PR. Renal cell cancer stage migration: Analysis of the National Cancer Data Base. Cancer. 2008; 113: 78-83.

39. Maloney E, Hwang JH. Emerging HIFU applications in cancer therapy. Int J Hyperthermia. 2015; 31: 302-9.

40. Jayson M, Sanders H. Increased incidence of serendipitously discovered renal cell carcinoma. Urology. 1998; 51: 203-5.

41. Heuer R, Gill IS, Guazzoni G, Kirkali Z, Marberger M, Richie JP. A critical analysis of the actual role of minimally invasive surgery and active surveillance for kidney cancer. Eur Urol. 2010; 57: 223-32.

42. Porpiglia F, Volpe A, Billia M, Scarpa RM. Laparoscopic versus open partial nephrectomy: Analysis of the current literature. Eur Urol. 2008; 53: 732-42, discussion 42-3.

43. Ritchie RW, Leslie T, Phillips R, et al. Extracorporeal high intensity focused ultrasound for renal tumours: a 3-year follow-up. BJU Int. 2010;106:1004-9.

44. Klatte T, Marberger M. High-intensity focused ultrasound for the treatment of renal masses: current status and future potential. Curr Opin Urol. 2009; 19: 188-91.

45. Ritchie RW, Leslie TA, Turner GD, et al. Laparoscopic high-intensity focused ultrasound for renal tumours: a proof of concept study. BJU Int. 2011; 107: 1290-6.

46. Wu F, Wang ZB, Chen WZ, Bai J, Zhu H, Qiao TY. Preliminary experience using high intensity focused ultrasound for the treatment of patients with advanced stage renal malignancy. J Urol. 2003; 170 (6 Pt 1): 2237-40.

47. Keane MG, Bramis K, Pereira SP, Fusai GK. Systematic review of novel ablative methods in locally advanced pancreatic cancer. World J Gastroenterol. 2014; 20: 2267-78.

48. Orsi F, Zhang L, Arnone P, et al. High-intensity focused ultrasound ablation: effective and safe therapy for solid tumors in difficult locations. AJR Am J Roentgenol. 2010; 195: W245-52.

49. Xiong LL, Hwang JH, Huang $\mathrm{XB}$, et al. Early clinical experience using high intensity focused ultrasound for palliation of inoperable pancreatic cancer. JOP. 2009; 10: 123-9.

50. Wu F, Wang ZB, Zhu H, et al. Feasibility of US-guided high-intensity focused ultrasound treatment in patients with advanced pancreatic cancer: initial experience. Radiology. 2005; 236: 1034-40.

51. Jang HJ, Lee JY, Lee DH, Kim WH, Hwang JH. Current and Future Clinical Applications of High-Intensity Focused Ultrasound (HIFU) for Pancreatic Cancer. Gut Liver. 2010; 4 Suppl 1: S57-61.

52. Li C, Zhang W, Fan W, Huang J, Zhang F, Wu P. Noninvasive treatment of malignant bone tumors using high-intensity focused ultrasound. Cancer. 2010; 116: 3934-42.

53. Bielack SS, Marina N, Bernstein M. High-intensity focused ultrasound (HIFU) is not indicated for treatment of primary bone sarcomas. Cancer. 2011;117: 2822; author reply 2822-3.

54. Liberman B, Gianfelice D, Inbar Y, et al. Pain palliation in patients with bone metastases using MR-guided focused ultrasound surgery: a multicenter study. Ann Surg Oncol. 2009; 16: 140-6.

55. Gianfelice D, Gupta C, Kucharczyk W, Bret P, Havill D, Clemons M. Palliative treatment of painful bone metastases with MR imaging--guided focused ultrasound. Radiology. 2008; 249: 355-63.

56. Catane R, Beck A, Inbar Y, et al. MR-guided focused ultrasound surgery (MRgFUS) for the palliation of pain in patients with bone metastases--preliminary clinical experience. Ann Oncol. 2007; 18: 163-7.

57. Jenne JW. Non-invasive transcranial brain ablation with high-intensity focused ultrasound. Front Neurol Neurosci. 2015; 36: 94-105.

58. Ghanouni P, Pauly KB, Elias WJ, et al. Transcranial MRI-Guided Focused Ultrasound: A Review of the Technologic and Neurologic Applications. AJR Am J Roentgenol. 2015; 205: 150-9.

59. Elias WJ, Huss D, Voss T, et al. A pilot study of focused ultrasound thalamotomy for essential tremor. N Engl J Med. 2013; 369: 640-8.

60. Magara A, Bühler R, Moser D, Kowalski M, Pourtehrani P, Jeanmonod D. First experience with MR-guided focused ultrasound in the treatment of Parkinson's disease. J Ther Ultrasound. 2014; 2: 11.

61. McDannold N, Clement GT, Black P, Jolesz F, Hynynen K. Transcranial magnetic resonance imaging- guided focused ultrasound surgery of brain tumors: initial findings in 3 patients. Neurosurgery. 2010; 66: 323-32; discussion 332.

62. Martin E, Jeanmonod D, Morel A, Zadicario E, Werner B. High-intensity focused ultrasound for noninvasive functional neurosurgery. Ann Neurol. 2009; 66: 858-61.

63. Al-Bataineh $\mathrm{O}$, Jenne J, Huber P. Clinical and future applications of high intensity focused ultrasound in cancer. Cancer Treat Rev. 2012; 38: 346-53.

64. Schroeder A, Kost J, Barenholz Y. Ultrasound, liposomes, and drug delivery: principles for using ultrasound to control the release of drugs from liposomes. Chem Phys Lipids. 2009; 162: 1-16.

65. Huang J, Xu JS, Xu RX. Heat-sensitive microbubbles for intraoperative assessment of cancer ablation margins. Biomaterials. 2010; 31: 1278-86.

66. Coussios CC, Farny CH, Haar GT, Roy RA. Role of acoustic cavitation in the delivery and monitoring of cancer treatment by high-intensity focused ultrasound (HIFU). Int J Hyperthermia. 2007; 23: 105-20.

67. Liu Y, Kon T, Li C, Zhong P. High intensity focused ultrasound-induced gene activation in solid tumors. J Acoust Soc Am. 2006; 120: 492-501.

68. Hancock HA, Smith LH, Cuesta J, et al. Investigations into pulsed high-intensity focused ultrasound-enhanced delivery: preliminary evidence for a novel mechanism. Ultrasound Med Biol. 2009; 35: 1722-36.
69. Huber PE, Pfisterer P. In vitro and in vivo transfection of plasmid DNA in the Dunning prostate tumor R3327-AT1 is enhanced by focused ultrasound. Gene Ther. 2000; 7: 1516-25.

70. Plathow C, Lohr F, Divkovic G, et al. Focal gene induction in the liver of rats by a heat-inducible promoter using focused ultrasound hyperthermia: preliminary results. Invest Radiol. 2005; 40: 729-35.

71. Deckers R, Rome C, Moonen CT. The role of ultrasound and magnetic resonance in local drug delivery. J Magn Reson Imaging. 2008; 27: 400-9. 